\title{
INTCare: A Knowledge Discovery based Intelligent Decision Support System for Intensive Care Medicine
}

\author{
Pedro Gago $_{* *}^{*}$ Manuel Filipe Santos ${ }_{* * * * *}^{* *}$ Álvaro Silva $^{* * *}$ \\ Paulo Cortez $^{* *}$ - José Neves ${ }^{* * * *}$ - Lopes Gomes
}

*Escola Superior de Tecnologia e Gestão do Instituto Politécnico de Leiria Morro do Lena, Alto Vieiro - Leiria - Portugal

pgago@estg.ipleiria.pt

**Departamento de Sistemas de Informação da Universidade do Minho

Campus de Azurém - Guimarães - Portugal

mfs,pcortez@dsi.uminho.pt

***Instituto de Ciências Biomédicas Abel Salazar

Porto-Portugal

a.moreira.silva@mail.telepac.pt

cardiologia.hgsa@mail.telepac.pt

**** Departamento de Engenharia Informática da Universidade do Minho

Braga - Portugal

jneves@di.uminho.pt

ABSTRACT. This paper introduces the INTCare system, an intelligent information system based on a completely automated Knowledge Discovery process and on the Agents paradigm. The system was designed to work in Hospital Intensive Care Units, supporting the physicians' decisions by means of prognostic Data Mining models. In particular, these techniques were used to predict organ failure and mortality assessment. The main intention is to change the current reactive behaviour to a pro-active one, enhancing the quality of service. Current applications and experimentations, the functional and structural aspects, and technological options are presented.

KEY WORDS: intelligent decision support, knowledge discovery, intensive care 


\section{Introduction}

The idea of conceiving Decision Making Support Systems (DMSS) making use of the Knowledge Discovery from Databases (KDD) and Agent-Based Systems paradigms, as a way to solve complex and dynamic problems, is not new (Fayyad et al., 1996; Weiss 1999; Santos 1999). However, a great part of these concepts (and architectures) need to be corroborated by real-world applications, in order to obtain a valuable feedback of its effective use. The intensive care medicine, due to its high volume and complexity of data, is a rich field to test this thesis.

DMSSs are computer based systems that support one or several phases of the individual, team organizational or inter-organizational decision making process (Forgionne et al., 2000). While DMSS constituted one of the most popular areas of research in information systems in the past, a closer look seems to indicate that the interest in DMSS is declining (Claver et al., 2000) or is not increasing at the rate that is expected (Vahidov et al., 2004). This is due to the requirements for decision support tools posed by new dynamic and complex environments (like the one in an ICU), demanding for situated and active DMSSs. A situated DMSS is similar to an open system in Artificial Intelligence (AI), a "view" that has been gaining popularity since the late 1980s (Suchman 1987). In a situated system the focus is on the interaction with its environment (Lueg et al., 1997), the behaviour is the combined result of its purpose and its interaction with the environment (Rosenschein et al., 1995).

Other concerns come from the extended Keen's agenda for DMSS research to the year 2007 (Shim et al., 2002), which postulates that researchers and developers should be more prescriptive about effective decision making by using intelligent systems and methods. The incorporation of Artificial Intelligence techniques, opened room for a new and more successful kind of DMSS, called intelligent decision making support systems (i-DMSS).

This paper introduces the INTCare, an i-DMSS based on the KDD and the Agent-Based paradigms, to support intensive care medical activities. In particular, the system assists the physicians' decision making by: (i) detecting action demanding conditions by continuously scanning automatically acquired data and applying the relevant model to predict next day failure of six systems (liver, respiratory, cardiovascular, coagulation, central nervous and renal); (ii) maintaining an up-to-date in-hospital death probability value used in end-of-life decision making and (iii) evaluating scenarios for the evolution of the condition of the patient, allowing physicians to compare the consequences of different medical procedures. The INTCare system is being tested in a real environment, the Intensive Care Unit (ICU) of Hospital Geral de Santo António (HGSA), Oporto, north of Portugal.

The following sections will introduce the problem definition and the state of the art, followed by the INTCare system formal description in terms of the Agent-Based paradigm. Then, the obtained results will be presented by means of their usability 
and efficiency. Next, a discussion will be set over the critical aspects of the INTCare system. Finally, the paper will be concluded, showing also perspectives of future work.

\section{Theoretical Background and Related Work}

\subsection{Decision Support Systems and Intensive Care}

In the beginning of the 80s, several expert systems were developed for medicine, such as MYCIN (Buchanan et al., 1984), CASNET (Kulikowski et al., 1982) and CADUCEUS (Pople 1985), just to name a few. A decade later, in the 90s, the common knowledge was that these expert systems, based on information retrieved from experts only, were not sufficient for the complex real-world problems (Lavrac et al., 1997). A shift was induced, where the main emphasis was the gathering of knowledge directly from the data, using intelligent data analysis. At the end of the 90s, this approach gained an increasing interest, especially in the medicine area, due to the high volume and complexity of the clinical data. In terms of intensive care medicine, the application of DM techniques (e.g. Artificial Neural Networks) is new, although possessing a huge potential (Hanson et al., 2001). However, it should be stressed that the number of studies were these techniques are applied in a real environment is very limited.

Everyday, doctors have to make decisions that seriously affect their patients. First, they make a diagnosis and then conceive therapeutic plans (e.g. prescribe treatments) in order to improve the patients' condition. However, ICU doctors have to make decisions that are even more challenging, such as the ones related to lifesupport treatments. Resource availability limitations force them to make sure that intensive care is applied only to those who are likely to benefit from it. Critical decisions include interrupting life-support treatments and writing do-not-resuscitate orders when intensive care is considered futile. In addition, the condition of ICU patients is such that doctors are not able to completely assess it and will benefit from having extra quality information available.

A synopsis of the current decision making process in the ICU of the HGSA is presented in Figure 1. This process can be seen as unfolding in four phases (Simon 1977) (Turban et al., 2004):

Intelligence - The medical and nursing staff collect physiological data regarding the patient's condition. Then, based on this information, the physicians evaluate the illness state to determine the pertinence of immediate action;

Design - Doctors conceive the possible therapeutic scenarios. Two main categories of scenarios can be considered: immediate action assumption (where there is a pressing need for intervention) and delayed group decision (guidelines for discharge or long term treatments). In the former case, if needed, the physician can 
consult internal or external colleagues. Delayed group decision choices include: patient's discharge, suspension of life-supporting treatment, writing of do-not resuscitate orders and applying a specific treatment;

Choice - After the formation of the alternatives and entwined with the previous stage, a final decision is taken (either individually or group based) and

Implementation - The corresponding therapeutic procedure is applied.

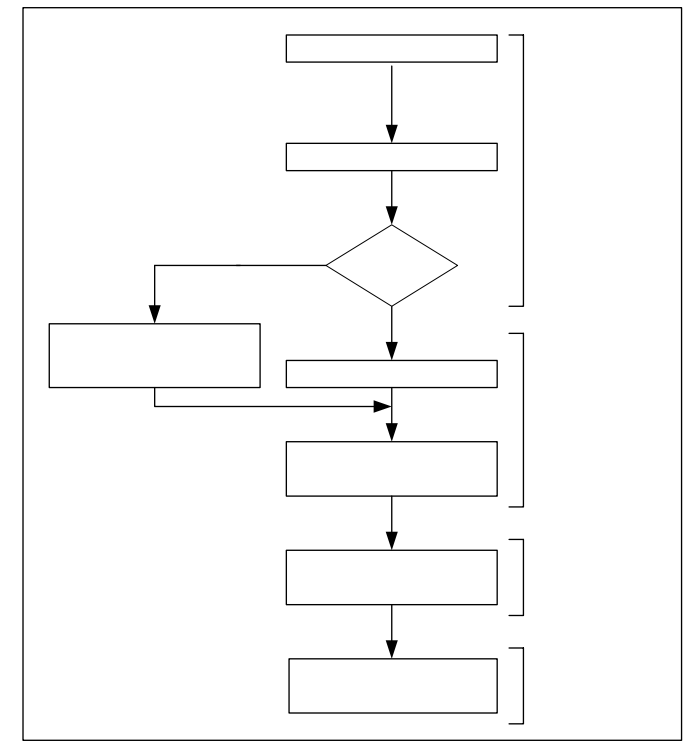

Figure 1. The HGSA ICU decision making process

At the current stage, doctors rely on their experience and on the available clinical data (e.g. patients chart sheets) to determine if there is a need for action. The same information is used when taking decisions about the course of action to be followed. No use is made of the vast amounts of data, which are automatically collected through bed-side monitors. To the authors' knowledge, although several prognostic scores have been developed (e.g. SAPS (Le Gall et al., 1993) or APACHE (Knaus et al., 1991), there are no intelligent DSS systems used in ICUs for end-of-life decision making. Yet, both patient and physicians could benefit if high-level, reliable and timely information is available. Hence, the INTCare system aims at filling this gap. The actual version of the system supports the intelligence phase by continuously scanning (sensing the environment) the available information in order to uncover action demanding situations. In addition, the design phase will be supported by evaluation of the consequences of the available therapeutic procedures. With the information gathered in the previous step, doctors can choose the procedure to 
follow. This is a particularly sensitive area as strong physician resistance is expected (Barnato et al., 2004). Finally, whenever allowed, INTCare can act on the environment controlling the equipment (e.g. infusion pumps).

\subsection{Knowledge Discovery from Databases and Data Mining}

The interest in Knowledge Discovery from Databases (KDD) and Data Mining (DM) arose due to the rapid emergence of electronic data management methods. In 1997, the Gartner Group suggested that DM is at the top five key technologies that will have a major impact in the industry within the next years (Lee et al., 2001). In effect, these techniques are now widespread and applications can be found in diverse areas, such as: marketing (Shaw et al., 2001) (Kim et al., 2004), banking (Hsieh 2004), manufacturing and production (Musaev 2004), brokerage and securities trading (Lam 2004), health care (Li et al., 2004), (Walker et al., 2004). References for other applications can be found in various publications, including (Turban et al,. 2004) and (Liao 2003).

Within the Medicine arena, huge databases, with large, complex and multisource information (e.g. text, images or numerical data), are commonplace. However, human experts are limited and may overlook important details. Furthermore, the classical data analysis (e.g. logistic regression) breaks down when such vast amounts of data are present. Hence, an alternative is to use automated discovery tools to analyze the raw data and extract high level information for the decision-maker (Hand et al., 2001).

An important step of the KDD process is related to the choice of the DM function, which will effect the selection of the DM algorithm (Fayyad et al., 1996). In ICUs, the most useful DM goals are:

- classification - labels a given set of attributes into one of several predefined classes (e.g. diagnosing a disease according to the patient's symptoms);

- regression - maps a data item into a real-value variable (e.g. estimation of the patient's heart rate); and

- clustering - searches for natural groupings of objects based on similarity measures (e.g. segmenting patients into clusters according to similar profiles).

The above goals may involve the application of Machine Learning algorithms such as Decision Trees (DT) (Quinlan 1986) (Lavrac et al., 1997), Learning Classifier Systems (LCS) (Lanzi et al., 2000, Santos 1999) and Artificial Neural Networks (ANN) (Haykin 1999) (Dybowski 2000). More recently, there has been an emergent DM research area that involves the use of ensembles for supervised learning, where a set of classification/regression models are combined in some way to produce an answer (Dieterich 1997). 


\subsection{Agent Based Systems}

The term agent is a metaphor allowing various definitions, interpretations and taxonomies. Actually, no one of them is universally accepted, despite this some positions are considered referential (Jennings et al., 1998; Nwama 1998; Weiss 1999; Ferber 1999). One of the most comprehensive definitions of agent was proffered by (Jennings et al., 1998) and is based on a strong and weak view. Applications of agents are widespread. Agents can be found in travel planning applications (Kim et al., 2004), e-commerce (Garcia-Serrano et al., 2004) and even as components of DMSSs (Hess et al., 2000).

In the context of this work, the AIMA definition prosecuted by (Russel et al., 2003) was adopted, stating that an agent is an entity capable of perceiving the environment and actuating on that environment. From a software engineering point of view, an agent is an abstraction that allows the construction of more complex systems designated by Agent-Based Systems or Agencies. Although, an Agency is typically conceptualized in terms of agents, the agents' structure can not be defined recursively (i.e. in terms of agents).

The requisites inherent of a situated and active system, in addition to the previous experience accumulated in the conception of agencies such as the DICE system, (Santos 1999) and the AIDA agency (Abelha 2003), dictated the adoption of the agent technology in the development of INTCare.

The various types of agents considered in the INTCare system are presented and discussed in the section 3 .

\subsection{Previous Work}

Artificial Intelligence techniques have been used in order to enhance DMSSs. One can find examples of ANN and genetic algorithms (Kim et al., 2004), DT (Tsang et al., 2004), fuzzy logic (Lin et al., 2004) and DM (Michalowsky et al., 2003) embedded in decision support systems. Likewise, agents are becoming commonplace as they provide a useful abstraction that facilitates systems conceptualization. An example of use of agents in the medical area is the AMPLIA system (Vicari et al., 2003). In an ICU setting the Guardian system (Hayes-Roth et al., 1998) monitors and makes diagnoses of intensive-care patients. Another system, the Rihad ICU Program (Chang et al., 1988) was used to predict death, having achieved high accuracies.

In our previous work, several DM models were applied to ICU data (Santos et al., 2002; Silva et al., 2003; Silva et al., 2004). These studies used off-line learning, where all data was stored first and then accessed repeatedly by the DM algorithms. The clinical data was collected during the EURICUS II research programme (Miranda et al., 2003), which involved a massive study in 42 ICUs from 9 countries 
during a period of 10 months, from 1998 to 1999. The database included thousands of daily records related to bedside measurements of critical ill patients, including features such as: the case mix - an information that remains unchanged during the patient's stay in the ICU (e.g. age or admission origin); the intermediate outcomes being triggered from four monitored biometrics (e.g. the systolic blood pressure or urine output); and the patient's state - based on daily organ failure scores (e.g. SOFA index (Vicent et al., 1998)) and the final outcome (death/no death). Intermediate outcomes are represented by the number of Critical Events per day - the number of daily occurrences of values out of the established limits for four physiologic variables that are monitored continuously. These four variables are Heart Rate, Systolic Blood Pressure, Oxygen Saturation and Urine Output. A group of clinical specialists determined the intervals considered normal for each one of these parameters.

This vast amount of information was modelled by:

- A LCS to predict the length of stay (short or prolonged) and the outcome (death/no death) of patients from the HGSA ICU, attaining an accuracy of $72 \%$ (Santos et al., 2002);

- A clustering framework to organ dysfunction diagnosis (Silva et al., 2003), where patients were segmented into clusters (using a Self-Organizing Map) and then each cluster was processed with the C.5 DT algorithm (the classification accuracies of the clusters ranged from $74 \%$ to $98 \%$ ); and

- An ANN approach (Silva et al., 2004), where easily acquired daily inputs (the intermediate outcomes) were fed into Multilayer Perceptrons, in order to predict the failure of six organic systems (e.g. liver or respiratory systems), achieving an overall accuracy of $70 \%$.

The outputs of the organ failure prediction models are measured by the SOFA (Sequential Organ Failure Assessment) score, which provides values within the range $\{0,1,2\}$ (normal function) and $\{3,4\}$ (failure). As for mortality assessment, the value one indicates in-hospital death while zero represents no in-hospital death predicted. Intermediate values can be read as probabilities of occurrence.

\section{INTCare System}

The INTCare is an agent based system, composed by several semi-autonomous agents in charge for the functionalities inherent to the system. Conceptually, it can be viewed as set of four subsystems (Figure 2): Data Entry, Knowledge Management, Inference and Interface. Formally, the INTCare system is defined as a tuple $\Xi \equiv\left\langle\mathrm{C}_{\mathrm{INTCare}}, \Delta_{\mathrm{INTCare}}, a_{\mathrm{pp}}, a_{\mathrm{cde}}, a_{\mathrm{dm}}, a_{\mathrm{pf},}, a_{\mathrm{mi}}, a_{\mathrm{dr}}, a_{\mathrm{pd}}, a_{\mathrm{sc}}, a_{\mathrm{int}}, a_{\mathrm{ic}}\right\rangle$, where: 
$C_{I N T C a r e}$ is the context and corresponds to a logical theory, represented as a triple $\langle L g, A x, \Delta\rangle$, where $L g$ stands for an extension to the language of programming logic, $A x$ is a set of axioms over $L g$, and $\Delta$ is a set of inference rules;

$\Delta_{I N T C a r e}$ is the set of bridge rules defining the interaction among the systems' components (the agents);

$a_{p p}, \ldots, a_{i c}$ are the system's agents.

This formalism corresponds to a logical framework, suitable to specify agentoriented systems based on the notion of context logic, and some properties of objectoriented design such abstraction, encapsulation, modularity and hierarchy (Santos 1999). In this work, the agents are represented as logical theories with a specific context (different agents may involve different contexts). Several agents (i.e. contexts) can be put together and be able to reason about the behaviour of the entire system as a (heterogeneous) logical theory. A set of special rules called bridge-rules is applied to provide the interface among agents and systems of agents. These rules describe the agents' reactions to events occurring in their environment. The agents include a set of event types, and a set of time points. Next, the overall system is described making use of this formalism, explaining it in some detail.

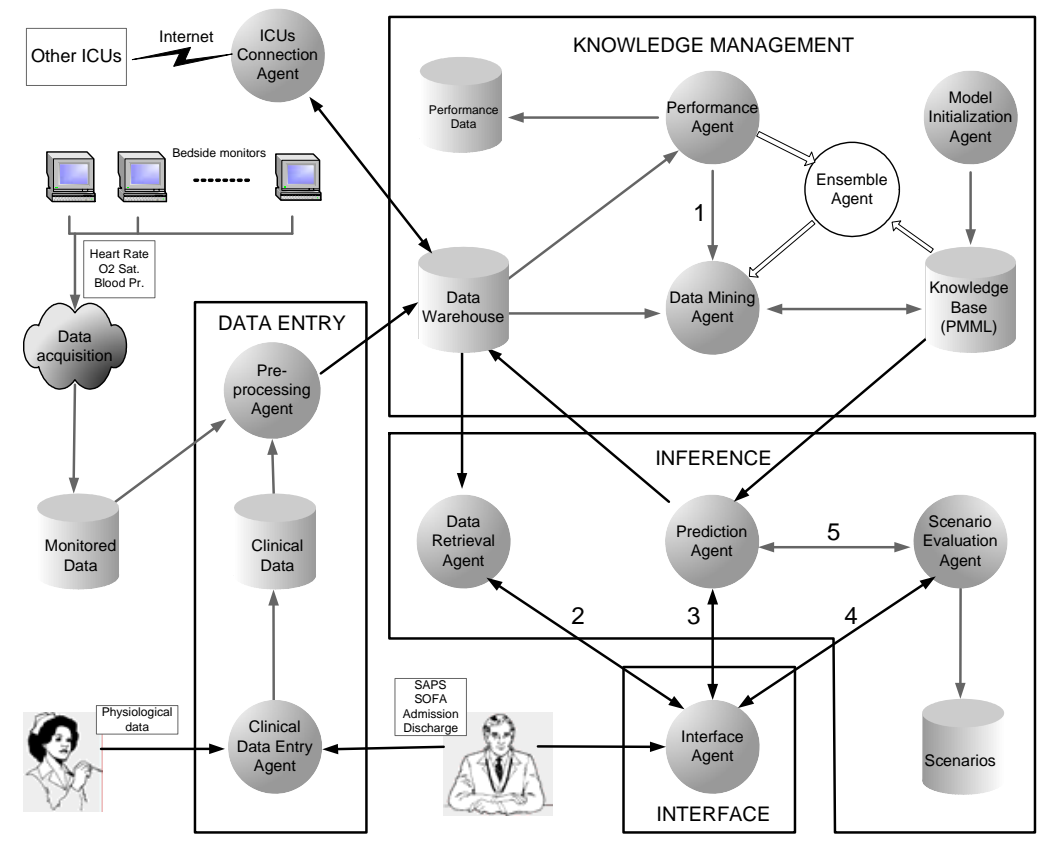

Figure 2. The INTCare system 


\subsection{Description of the Agents}

The INTCare agents follow the subordinated architecture (Weiss 1999), they are social computational entities, semi-autonomous, reactive, with internal-state, and pre-defined goals incited by the system creators, whose activity contributes to the goal of the overall system. In a technical perspective the INTCare agents are high granularity objects aggregating a great number of capacities.

The social model is static, pre-defined, and incorporated into the system by the developers. The intelligent behaviour, the accuracy, the robustness, the flexibility and efficiency of this kind of system emerges from the agents and their interaction. The overall behaviour is the combined result of its purpose and its interaction with the environment (Vahidov et al., 2004). All agent interaction is defined using events. The next lines describe the agents' functionalities and the associated events:

Clinical Data Entry $\left(a_{c d e}\right)$ agent is responsible for the capture of clinical data from the medical and nursing staff;

Pre-Processing $\left(a_{p p}\right)$ agent is responsible for the correct linking of all the values in order to create a valid (even if limited in scope) medical record for the patient. It proceeds with the copy of the values entered by the medical and nursing staff (or recorded via the bedside monitors), examines them and derives new fields, if necessary (such as Critical Events);

Data Mining $\left(a_{d m}\right)$ agent belongs to the Knowledge Management sub-system and is responsible for the retrieval of the relevant data (read_dw) in order to make possible the application of artificial intelligence algorithms to train new models (train), whenever requested by the Performance $\left(a_{p f}\right)$ agent, storing them into the Knowledge Base (update_models). After training, the models are stored in the Knowledge Base, using the Predictive Markup Model Language (PMML), which is a XML based model description language (URL1 2004);

Performance $\left(a_{p f}\right)$ agent continually consults, in a proactive way, the the Data Warehouse for updates that allow statistics collection (e.g. discharge data that may or may not confirm a prediction made), as a base to calculate a set of assessment parameters maintained in the Performance Database. The evaluation metrics include classification accuracy, sensitivity and specificity values (Silva et al 2004). These statistics are updated every time new relevant information is collected. Whenever the collected statistics show that the performance has fallen bellow a predefined threshold (a configuration parameter) a new model is requested in order to allow the replacement of the poor performing one (message_dm);

Model Initialization $\left(a_{m i}\right)$ agent populates the Knowledge Base with the models obtained from off-line training. This agent is currently used only when first starting INTCare. As external models may be represented in a PMML version that is not used in INTCare, this agent must perform a conversion before loading the models into the Knowledge Base; 
Data Retrieval $\left(a_{d r}\right)$ agent is an information agent, whose only objective is that of retrieving from the Data Warehouse the information requested by the interface agent (get_data) and returning it (send_data) ;

Prediction $\left(a_{p d}\right)$ agent answers user questions by applying the adequate models (get_models, predict) in the Knowledge Base to the data stored in the Data Warehouse. Next, results are sent back to the calling agent (send_data) and if the calling agent is the interface agent, the prediction made is recorded in the Data Warehouse (update_dw);

Scenario Evaluation $\left(a_{s c}\right)$ agent makes it possible to the doctor to create and evaluate what-if scenarios. After receiving the data from the interface agent, the scenario evaluation agent requests a forecast from the prediction agent (re_prediction), the scenario is then stored in the Scenarios Database (store_scenario) and the result is sent back to the interface agent (send_result).

Interface $\left(a_{\text {int }}\right)$ agent allows (web-based) interaction with the system by providing an easy way for doctors to request prognostics and evaluate scenarios. Whenever new data is needed, this agent messages the data retrieval agent (message_dr). In Figure 3 a screen shot of the prediction window (re_prediction) is presented where it is possible to consult the prognostics for a single patient and at the same time compare the past SOFA predictions with the real values. A holistic view of the patient is supported by presenting in the same chart the information regarding the diverse organic systems. Also, the values predicted for the next day are shown providing information that may suggest the need for immediate action. For the past days, the actual SOFA score is shown, together with the value predicted for the next day. It is possible to consult the confidence levels associated with the prediction values (confidence button);

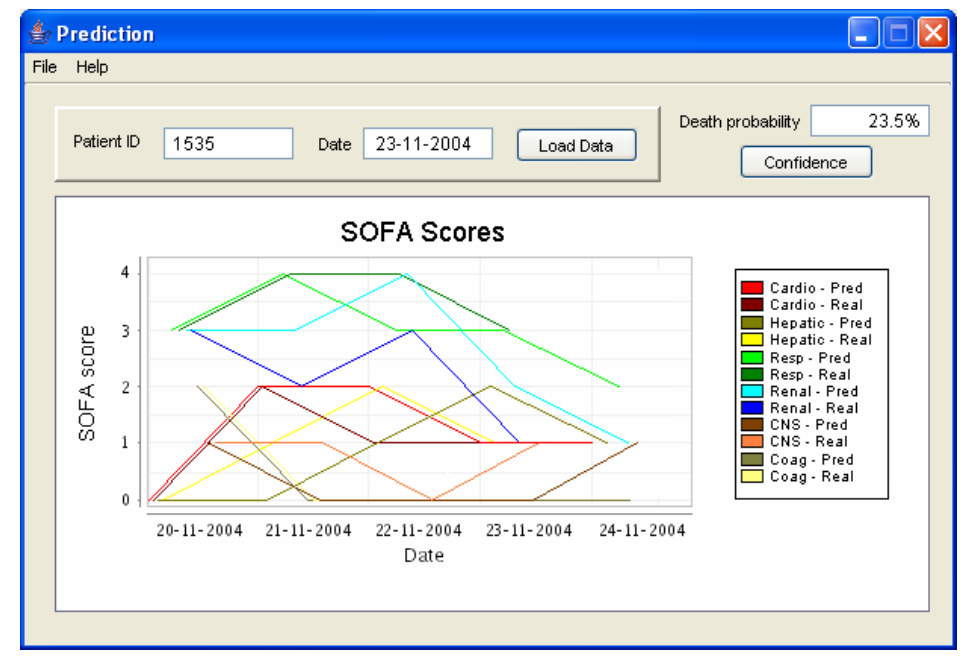

Figure 3. INTCare User Interface 
Ensemble agent intended to enhance predictive performance by combining several models (e.g. using a majority voting scheme) in order to produce an answer.

Connection Agent $\left(a_{i c}\right)$ is in charge for the knowledge interchange among the various instances of the INTCare system running in the ICUs.

The interaction among the INTCare's agents, is represented in terms of the bridge rules $\Delta_{\text {INTCare }}$ described in Table 1 (where $t$ stands for the system's time cycle). The numbers to the left of the rules were used in Figure 1 to label the arrows representing the interactions.

\begin{tabular}{|c|c|c|}
\hline & Bridge-Rule & Description \\
\hline 1 & $\begin{array}{l}a_{p f}: \text { occurs }(\text { message_dm, } t) \\
a_{d m}:[\text { occurs }(\text { read_dw, } t) \wedge \\
\text { occurs }(\text { train, } t) \wedge \\
\text { occurs }(\text { update_models, } t)]\end{array}$ & $\begin{array}{l}\text { When the Performance agent detects the need for the } \\
\text { replacement of a model, the Data Mining agent is } \\
\text { messaged, reads the available data in the Data } \\
\text { Warehouse, trains a new model and finally saves it } \\
\text { into the Knowledge Base. }\end{array}$ \\
\hline 2 & $\begin{array}{c}a_{\text {int }}: \text { occurs }(\text { message_dr, } t) \\
a_{d r}:\left[\text { occurs }\left(g e t \_d a t a, t\right) \wedge\right. \\
\text { occurs }(\text { send_data, } t)]\end{array}$ & $\begin{array}{l}\text { When triggered by the Interface agent, the Data } \\
\text { Retrieval agent gets the patient's data from the Data } \\
\text { Warehouse and sends it back to the Interface agent. }\end{array}$ \\
\hline 3 & $\begin{array}{c}a_{\text {int }}: \text { occurs }\left(r e \_p r e d i c t i o n, t\right) \\
a_{p d}:[\text { occurs }(\text { get_models, } t) \wedge \\
o c c u r s(\text { predict } t) \hat{\wedge} \\
\text { occurs }(\text { send_data, } t) \wedge \\
o c c u r s(\text { update_dw, } t)]\end{array}$ & $\begin{array}{l}\text { When the Interface agent requests a forecast, the } \\
\text { Prediction agent gets the adequate model from the } \\
\text { Knowledge Base applies it and finally sends the } \\
\text { results back to the Interface agent and updates the } \\
\text { Data Warehouse. }\end{array}$ \\
\hline 4 & $\begin{array}{c}\frac{a_{\text {int }}: \text { occurs }\left(r e \_s c e n a r i o, t\right)}{a_{\text {sce }}:\left[\text { occurs }\left(r e \_p r e d i c t i o n, t\right)\right.} \wedge \\
\text { occurs }(\text { store_scenario, } t) \wedge \\
\text { occurs }(\text { send_result, } t)]\end{array}$ & $\begin{array}{l}\text { When the Interface agent requests a scenario } \\
\text { evaluation it sends the necessary data to the Scenario } \\
\text { Evaluation agent that then requests the forecast from } \\
\text { the Prediction agent, stores the scenario in the } \\
\text { Scenarios Database and finally returns the scenario } \\
\text { results to the Interface agent. }\end{array}$ \\
\hline 5 & $\begin{array}{c}a_{s c e}: \text { occurs }\left(r e \_p r e d i c t i o n, t\right) \\
a_{p d}:[\text { occurs }(\text { get_models, } t) \wedge \\
o c c u r s(\text { predict } t) \wedge \\
o c c u r s(\text { send_data, } t)]\end{array}$ & $\begin{array}{l}\text { When the Scenario Evaluation agent requests a } \\
\text { forecast, the Prediction agent gets the adequate } \\
\text { model(s) from the Knowledge Base applies it and } \\
\text { finally sends the results back to the requesting agent. }\end{array}$ \\
\hline
\end{tabular}

Table 1. INTCare’s bridge-rules.

\section{Deployment and Experimentation}

INTCare was programmed using the Visual Basic . Net ${ }^{1}$ environment, PMML (to represent the models in the Knowledge Base), the Xelopes library (prudsys 2004)

${ }^{1}$ All other trademarks or registered trademarks stated in this manual are property of the respective owners. 
and Jbuilder. The system is currently under use in the ICU of the HGSA in Oporto, Portugal. This unit contains ten monitored beds, treating around 400 patients each year. Regarding the deployment, it was planned to occur in three separate steps. First, the system started to be used with limited data (acquired from a single bedside monitor). This allowed for a less invasive introduction in the operating environment as only two doctors were involved at this time. The next step extends data acquisition to the ten available monitors and widespread use of the system by the doctors working in this ICU. Finally, INTCare will be extended to two other ICUs within the same region of influence.

The current configuration includes one bed-side monitor and two personal computers running Windows. One of the PCs is dedicated to data collection, the other is where INTCare is available for use by the doctors. A TCP/IP network allows communication between the diverse components. All data acquisition software runs under Windows.

Experimentation has started as planned, with data acquisition from one bed-side monitor, therefore the information is available for a limited number of patients and consequently the results can not be considered statistically meaningful. The available results are given in Table 2 .

\begin{tabular}{cccc}
\hline Prediction & Accuracy & Sensitivity & Specificity \\
\hline In-hospital death & 80.8 & 60.0 & 85.7 \\
\hline Respiratory & 60.5 & 54.5 & 68.8 \\
\hline Coagulation & 94.7 & 80.0 & 97.0 \\
\hline Hepatic & 86.8 & 70.0 & 100 \\
\hline Cardiovascular & 94.7 & 0.0 & 97.3 \\
\hline Central N System & 100 & n.a. & 100 \\
\hline Renal & 97.4 & 96.2 & 100 \\
\hline
\end{tabular}

Table 2. Prediction results (\%)

Although the results attained for organ failure prediction, in some organic systems (e.g. renal, cardiovascular and central nervous system) can be considered excellent, it should be stressed that they are not meaningful due to the low number of patients considered. This lack of data also prevented the retraining of the models. We could not find other SOFA prediction systems in order to compare our results. Our in-hospital death prediction results are comparable to those of RICP even though INTCare always gives a death/no-death result whereas RICP reports "death" or "outcome unknown", thus reducing the number of false positive answers. Nevertheless, the feedback received from the doctors in relation to the system usage is positive, the predictions were considered "interesting". In the future, the system is expected to play a significant role in the ICU decision procedures. 


\section{Discussion and Technological Impact}

Currently, intensive care decision making is based on the individual doctor's previous empirical experience. In the majority of ICUs, patients are continuously monitored with several biometric sensors. Yet, this information is not fully taken into the decision process. This scenario changes with the introduction of the INTCare system, where all this data is stored and made available. Furthermore, the INTCare is enriched with DM prognostic models, which can provide forecasts of both intermediate and final outcomes. In addition, the physicians may also evaluate different scenarios; i.e. to test what would occur if a given condition is changed.

Previous results (Silva et al., 2003, Silva et al., 2004) show the reliability of the DM prognostic models. Nevertheless, introduction of model-based outcome prediction for has to overtake some problems, including: reliability, availability and physician resistance (Barnato et al., 2004). In INTCare, the availability problem is partly solved by using automatic data acquisition techniques. Reliability is expected to increase when the ensemble agent is completed. Physician resistance is expected to soften as the system is to be used as a support tool and its performance is expected to improve with the feedback taken from the physicians.

A computer based system has other advantages such as not being subject to between-physician variability in prognostic skill or beliefs regarding the construct of "futility". Furthermore model-based predictions may be more equitable because they do not incorporate value-based judgments regarding the "worth" of one life over another (Barnato et al., 2004). The usability (interaction with humans) and technology acceptance results are presented in Table 3:

\begin{tabular}{lll}
\hline \multicolumn{1}{c}{ Situation } & \multicolumn{1}{c}{ Present } & \multicolumn{1}{c}{ Expected } \\
\hline $\begin{array}{l}\text { access to patient's } \\
\text { records }\end{array}$ & paper records & digital records \\
$\begin{array}{l}\text { comparing present and } \\
\text { old patient's data } \\
\text { decision making }\end{array}$ & $\begin{array}{l}\text { someone has to find the } \\
\text { right file - manual search } \\
\text { during a doctors meeting }\end{array}$ & $\begin{array}{l}\text { computer aided } \\
\text { INTCare is another expert } \\
\text { in the doctors meeting } \\
\text { scenario evaluation }\end{array}$ \\
\hline
\end{tabular}

Table 3. Expected impact of the system

\section{Conclusions and further work}

This paper presented an Agent-Based System called INTCare, for ICU real time Decision Support on clinical actuation using a KDD/DM approach for automatic knowledge acquisition. The architecture and functionalities where defined in terms of a formal framework. The major contributions of this approach are: 
- $\quad$ The system is being tested in a real-world environment which means that it is tailored to the physician needs;

- A better overall predictive performance is enabled by the competition and combination of heterogeneous DM algorithms;

- $\quad$ The system implements all the KDD steps in an automatic way, leading to an autonomous situated Intelligent Information System;

- The Data Mining process is enhanced by the construction of a Data Warehouse which is automatically filled by a Pre-Processing agent.

The INTCare system is currently being tested in the ICU of HGSA. The preliminary results even promising are not absolutely credible due the limited number of cases recorded. Further experiments will consider a greater volume of monitored data and cases analysing its impact in terms of on-line and incremental learning. Efforts will be made towards a major performance through the system tuning, the ensemble agent exploitation, involving more ICUs (from the same hospital and from other hospitals in Portugal or even from other European countries) and the inclusion of new variables in the prediction models (e.g. data regarding patient ventilation). Finally we will pay attention to the therapeutic procedures, covering the choice and design phases of the decision making process by suggesting possible actions and predicting their consequences. It is planned the control of medical equipment (e.g. infusion pumps) automating the implementation phase.

\section{References}

Abelha S., Machado J., et al. (2003) Agency for Integration, Diffusion and Archive of Medical Information, in proceedings of the IASTED International Conference on Artificial Intelligence and Applications, Benalmádena, Spain, September

Barnato A. and Angus D. (2004) Value and role of intensive care unit outcome prediction models in end-of-life decision making. Crit Care Clin 20:345-362

Buchanan B.G. and Shortliffe E.H. (1984) Rule-Based Expert Systems: The MYCIN Experiments of the Stanford Heuristic Programming Project. Reading, MA: AddisonWesley

Chang R., Jacobs S., Lee B. (1988) Predicting outcome among intensive care unit patients using computerised trend analysis of daily Apache II scores corrected for organ system failure. Intensive Care Med 14(5): 558-566

Claver E., Gonzales R., Llopis J. (2000) An analysis of research in information systems (1981-1997), Information and Management 37 181- 195

Dietterich T. (1997) Machine Learning Research: Four Current Directions. AI Magazine 18(4) $97-136$

Dybowski R. (2000) Neural Computation in Medicine: Perspectives and Prospects. In: Malmgreen et al. (eds) Proceedings of the ANNIMAB-1 Conference (Artificial Neural Networks in Medicine and Biology). Springer, Berlin Heidelberg New York, pp 26-36

Essex-Sorlie D. (1995) Medical Biostatistics \& Epidemiology: Examination \& Board Review. McGraw- Hill/Appleton \& Lange, International edition 
Fayyad U., Piatesky-Shapiro G., Smyth P. (1996) From Data Mining to Knowledge Discovery: An Overview. In Fayyad et al. (eds) Advances in Knowledge Discovery and Data Mining. AAAI Press / The MIT Press, Cambridge MA, pp 471-493

Ferber J. (1999) Multi-Agent Systems - An Introduction to Distributed Artificial Intelligence, Addison-Wesley

Forgionne G.A., Mora M., Cervantes F., Kohli R (2000) Development of integrated decision making support systems: a pratical approach. In Proceedings of the AIS Conference, Long Beach CA, pp 2132-2134

Garcia-Serrano A.M., Martinez P., Hernandez J.Z. (2004) Using AI techniques to support advanced interaction capabilities in a virtual assistant for e-commerce. Expert Systems with Applications 26(3): 413-426.

Hand D., Mannila H., Smyth P.(2001) Principles of Data Mining. MIT Press, Cambridge, MA

Hanson C., Marshall B. (2001) Artificial intelligence applications in the intensive care unit. Crit Care Med 29(2) 427-435

Hayes-Roth B., Larsson J.E. (1998) Guardian: an intelligent autonomous agent for medical monitoring and diagnosis. IEEE Intelligent Syst 1998(January/February):58-64

Haykin S. (1999) Neural Networks - A Compreensive Foundation ( $2^{\text {nd }}$ Edition). PrenticeHall, New Jersey

Hess T.J., Rees, L.P., Rakes T.R. (2000) Using autonomous software agents to create next generation of decision support systems. Decision Sciences 31 (1) 1-31

Hsieh N.C. (2004) An integrated data mining and behavioral scoring model for analyzing bank customers. Expert Systems with Applications 27(4): 623-633

Jennings R., Wolldridge J. (1998) Agent Technology Foundations, Applications and Markets. Springer, Berlin Heidelberg New York

Knaus W.A. et al. (1991) The APACHE III prognostic system. Risk prediction of hospital mortality for critically ill hospitalized adults Chest 1991 100: 1619-1636

Yim H.S., Ahn H.J., Kim J.W., Park S.J. (2004) Agent-based adaptive travel planning system in peak seasons. Expert Systems with Applications 27(2): 211-222

Kim Y. and Street W.N. (2004) An intelligent system for customer targeting: a data mining approach. Decision Support Systems 37(2): 215-228

Kohavi R., Provost F. (1998) Glossary of terms. Machine Learning, 30(2/3):271-274.

Kulikowski C.A., Weis S.M. (1982) Representation of expert knowledge for consultation: the CASNET and EXPERT projects, in: P. Szolovits (ed.), Artificial Intelligence in Medicine, Westview Press, Boulder, 21-56.

Lam M. (2004) Neural network techniques for financial performance prediction: integrating fundamental and technical analysis. Decision Support Systems 37(4): 567-581

Lanzi P., Solzman W., Wilson S. (2000) Learning Classifier Systems From Foundations to Applications. Lecture Notes in Artificial Intelligence 1813, Springer, Berlin Heidelberg New York

Lavrac N., Keravnou E., Zupan B. (1997) Intelligent Data Analysis in Medicine and Pharmacology. Kluwer, Boston

Le Gall J.R., Lemeshow S., Saulnier F. (1993) A new Simplified Acute Physiology Score (SAPS II) based on a European/North American multicenter study. JAMA 270: 29572963

Lee S. and Siau K. (2001) A review of data mining techniques. Industrial Management \& Data Systems, 101 (1): 41-46, MCB University Press

Li L., Tang H. , et al. (2004) Data mining techniques for cancer detection using serum proteomic profiling. Artificial Intelligence in Medicine 32(2): 71-83 
16 Title of the journal. Volume $\mathrm{X}$ - no X/2002

Lin, C.. and Hsieh P-J.(2004). "A fuzzy decision support system for strategic portfolio management." Decision Support Systems 38(3): 383-398

Liao S-h (2003) Knowledge management technologies and applications-literature review from 1995 to 2002. Expert Systems with Applications 25(2): 155-164

Lueg C., Pfeifer R. (1997) Cognition, situatedness, and situated design. Second International Conference on Cognitive Technology, IEEE Computer Society, Aizu, Japan

Michalowski W., Rubin S., et al. (2003) Mobile clinical support system for pediatric emergencies. Decision Support Systems 36(2): 161-176

Miranda D., Nat R., Nijk A., Schaufeling W., Lapichino G. (2003) Nursing activities score. Crit Care Med 31:374-382

Musaev A. (2004) Analytic information technologies in oil refinery. Expert Systems with Applications 26(1): 81-85

Pople H.E. (1985) Evolution of an Expert System: from INTERNIST to CADUCEUS. In: Artificial Intelligence in Medicine (ed. by I. De Lotto and M. Stefanelli), 179-208. Elsevier Science Publisher, Amsterdam.

prudsys AG. (2004) Xeli's Intro - Introduction to XELOPES Version 1.2.3 (Xelopes Users Manual)

Quinlan J. (1986) Induction of Decision Trees. Machine Learning 1 (1) 81-106

Rosenschein S.J., Kaelbling L.P. (1995) A situated view of representation and control, Artificial Intelligence $73(1-2)$ 149- 173

Russel S. and Norvig P. (2003) Artificial Intelligence - A Modern Approach $2^{\text {nd }}$ Edition. Prentice Hall, New Jersey.

Santos, M.F. (1999) Sistemas de Classificação em Ambientes Distribuídos. Ph.D. thesis, Umiversidade do Minho

Santos M.F., et al. (2002) Augmented Data Mining over Clinical Databases: Using Learning Classifier Systems. In: Proceedings of the Fourth International Conference on Enterprise Information Systems (ICEIS 2002), INSTICC, 512-516

Shim J.P. et al. (2002) Past, Present, and future of decision support technology. Decision Support Systems (33) 111-126

Shaw M.J., Subramaniam C., Gek Woo T., Welge, M.E. (2001) Knowledge management and data mining for marketing. Decision Support Systems 31(1): 127-137.

Silva A., Pereira J., Santos M.F., Gomes L., Neves J. (2003) Organ failure prediction based on clinical adverse events: a cluster model approach. In: Hamza MH (ed) Proceedings of the third IASTED International Conference on Artificial Intelligence and Applications (AIA 2003). ACTA Press

Silva A., Cortez P., Santos M.F., Gomes L., Neves J. (2004) Multiple organ failure diagnosis using adverse events and neural networks. In: Seruca I et al. (eds) Proceedings of the $6^{\text {th }}$ International Conference on Enterprise Information Systems (ICEIS 2004) Vol II, INSTICC, pp 401-408

Simon H. (1977) The New Science of Management Decisions, Rev. ed. Englewood Cliffs. Prentice Hall, New Jersey

Suchman L. (1987) Plans and Situated Actions: The Problem of Human -Machine Communication, Cambridge Univ. Press, Cambridge, MA

Tsang E., Yung P., et al. (2004) EDDIE-Automation, a decision support tool for financial forecasting. Decision Support Systems 37(4): 559-565

Turban E., Aronson J., Liang T. (2004) Decision Support Systems and Intelligent Systems, Prentice Hall, New Jersey 
Vahidov R., Kersten G. (2004) Decision station: situating decision support systems. Decision Support Systems (38) 283-303

Vicari R.M., Flores C.D., et al. (2003) A multi-agent intelligent environment for medical knowledge. Artificial Intelligence in Medicine 27(3): 335-366

Vincent J-L., De Mendonça A., Cantraine F., et al.( 1998) Use of the SOFA score to assess the incidence of organ dysfunction/failure in intensive care units: results of a multicenter, prospective study. Crit Care Med 26: 1793-1800

Walker R. , Smith P.B., et al. (2004) Data mining of gene expression changes in Alzheimer brain. Artificial Intelligence in Medicine 31(2): 137-154

Weiss G. (1999) Multiagent Systems A Modern Approach to Distributed Artificial Intelligence, MIT Press, Cambridge MA

URL1 2004 - http://soleunet.ijs.si/website/other/DMiner_and_PMML.pdf 